\title{
Nicorandil: a useful adjunct for proteinuria?
}

The ATP-sensitive potassium $\left(\mathrm{K}_{\mathrm{ATP}}\right)$ channel agonist nicorandil could be a beneficial addition to standard antiproteinuric treatment, according to researchers in Taiwan. "This finding might open new windows to treating proteinuric patients who are refractory to current therapy," says co-investigator Tsung-Ming Lee.

Renin-angiotensin system inhibitors do not stop the progression of renal disease in patients with proteinuria; therefore, alternative therapies are needed. As $\mathrm{K}_{\text {ATP }}$ channel agonists have antiproteinuric effects in animal models of renal disease, Lee and colleague Nen-Chung Chang investigated whether nicorandil would further reduce proteinuria in individuals with well-controlled hypertension who were receiving valsartan.

The researchers randomly assigned 136 patients to receive adjunctive treatment with placebo, the nitric-oxide donor isosorbide dinitrate ( $30 \mathrm{mg}$ per day) or nicorandil ( $15 \mathrm{mg}$ per day) for 6 months. Kidney disease was an exclusion criterion.

After 6 months, nicorandil-treated patients showed significant decreases in proteinuria, whereas proteinuria remained stable in patients treated with isosorbide dinitrate or placebo. Urinary levels of endothelin 1 and retinol-binding protein (a marker of proximal tubule function) also decreased with nicorandil.

Since nitric-oxide release seemed not to have antiproteinuric effects and nicorandil did not alter hemodynamic parameters, Lee and Chang conclude that the molecule might act by reducing renal endothelin 1 synthesis and improving tubular function.

\section{Rebecca Ireland}

Original article Lee, T. M. \& Chang, N. C. Effect of nicorandil on proteinuria in well controlled hypertensive patients. J. Hypertens. 27, 618-625 (2009). 